\title{
Enhanced Nanohydroxyapatite Formation Using a Hydrolyzed Gelatin
}

\author{
Ding Ding, ${ }^{1}$ Tengfei Yu, ${ }^{2}$ Weijie Zhang, ${ }^{1}$ Wei Liu, ${ }^{1}$ and Yaqin Huang ${ }^{1}$ \\ ${ }^{1}$ State Key Laboratory of Chemical Resource Engineering, Beijing Laboratory of Biomedical Materials, \\ Beijing University of Chemical Technology, Beijing 100029, China \\ ${ }^{2}$ Department of Ultrasound, Nanlou Clinic Division, Chinese People's Liberation Army General Hospital, Beijing 100853, China
}

Correspondence should be addressed to Yaqin Huang; huangyaqin9@sina.com

Received 2 August 2015; Revised 12 October 2015; Accepted 28 October 2015

Academic Editor: Andrea Falqui

Copyright (C) 2015 Ding Ding et al. This is an open access article distributed under the Creative Commons Attribution License, which permits unrestricted use, distribution, and reproduction in any medium, provided the original work is properly cited.

\begin{abstract}
Hydroxyapatite (HAP) nanoparticles were synthesized at room temperature using hydrolyzed gelatin (HG) as an additive. The crystallinity of HAP particles can be effectively controlled by adjusting the concentration of HG additive and the $\mathrm{pH}$ value of reaction system. The synthesized product was analyzed by Fourier transform infrared (FTIR) spectrometer, energy dispersive spectrometer (EDS), X-ray diffractometry (XRD), and transmission electron microscope (TEM). Meanwhile, it was proved that, with HG additive, HAP can be prepared with the calcium-phosphorus ratio of not only 1.67 but also 1.50. Conventionally, HAP particles need to be synthesized at high temperature with $\mathrm{iCa} / \mathrm{P}=1.67$. Therefore, we believe that this study provides a novel strategy for the preparation of nano-HAP.
\end{abstract}

\section{Introduction}

Hydroxyapatite (HAP) has attracted much attention recently in the field of bioengineering [1], especially as a bone substitute material due to its biological compatibility and bone conduction. A variety of methods have been developed to synthesize HAP nanoparticles [2-7], such as phase transformation, chemical deposition, hydrothermal, and microemulsion methods. Researchers found that HAP crystal growth depended greatly on the properties of the starting solution, and the morphology of the HAP crystals could be affected by the initial $\mathrm{Ca} / \mathrm{P}$ ratio $(\mathrm{iCa} / \mathrm{P})$. Conventionally, pure $\mathrm{HAP}$ particles can be synthesized at the higher temperature with $\mathrm{iCa} / \mathrm{P}=1.67$ [8]. Compared to these artificially synthesized particles, HAP particles obtained from natural bone exhibit better mechanical and biological properties [9]. This biomineralization process involves interactions between organic natural polymer and inorganic matter [10]. The functional groups of organic natural polymer can interact with calcium ions and promote nucleation via molecular recognition. The interaction between functional groups of organic natural polymer and ions in the solution decreases the nucleation activation energy to some extent. Therefore, it is possible to obtain HAP nanoparticles at room temperature. Moreover, the addition of organic natural polymer can also promote the formation of structural organization through polymer-ion interactions. Therefore, we have developed a new strategy in this work to obtain HAP nanoparticles using some principles of biomineralization.

Animal bone is an organic/inorganic composite with collagen and HAP. Previous studies reported that collagen promoted the formation of HAP in collagen fibrils scaffold [11]. Collagen is a kind of nature polymer. This inspired us to design a new way to prepare the nano-HAP using the hydrolyzed gelatin $(\mathrm{HG})$ as a multifunctional additive. Our procedure for preparation of the nano-HAP is shown in Scheme 1.

The rationales under our design include the following. (1) HG is the degradation product of collagen. It may enhance the formation of HAP as it has the similar amino acid composition to collagen. (2) HG is soluble in cold water, which facilitates HAP purification. (3) It is expected that the terminal functional groups, such as carboxyl group, hydroxyl oxygen atoms, and oxygen atoms from hydroxyl and carbonyl 


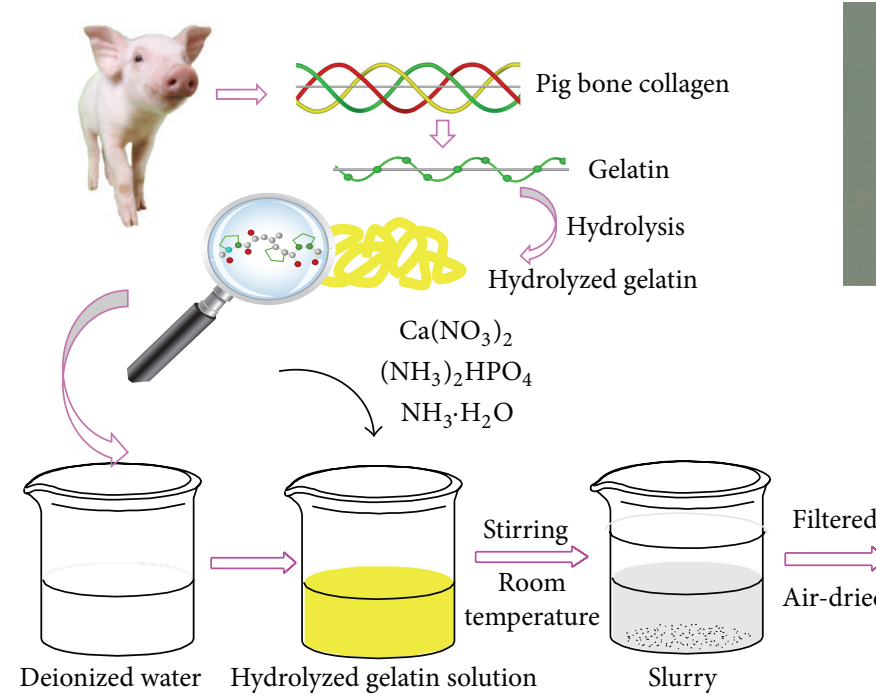

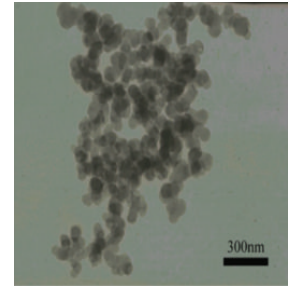

TEM images of the sample with HG

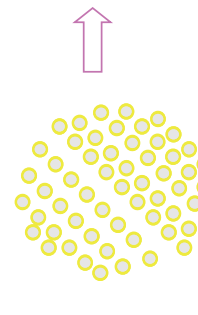

White powder

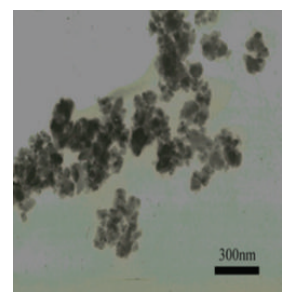

TEM images of the calcined sample at $700^{\circ} \mathrm{C}$

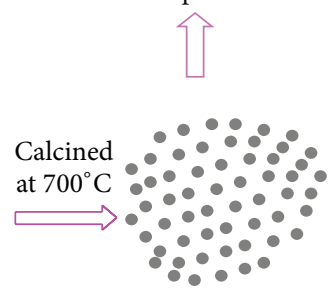

Nanohydroxyapatite

Hydrolyzed gelatin

Hydroxyapatite

Scheme 1: Preparation of the nanohydroxyapatite using hydrolyzed gelatin as an additive.

groups $[7,11]$, in $\mathrm{HG}$ molecules can interact with calcium ions. This kind of interactions may promote the formation of HAP and the mediation of the morphology in nano-HAP particles. (4) This additive may also reduce the aggregation of the particles and induce the formation of certain morphology due to the three-dimensional conformation formed by polypeptide chains in the HG solution. (5) HG is also commercially available and environmentally friendly. This report provides a detailed description of this novel nano-HAP synthesis using HG as a functional additive.

\section{Experimental}

2.1. Preparation. A certain amount of $\mathrm{HG}(2 \mathrm{wt} \%, 5 \mathrm{wt} \%$, and $10 \mathrm{wt} \%)$ was firstly dissolved in $\mathrm{Ca}\left(\mathrm{NO}_{3}\right)_{2}$ aqueous solution, and then $\left(\mathrm{NH}_{4}\right)_{2} \mathrm{HPO}_{4}$ aqueous solution was added slowly, until the ratio of $\mathrm{n}(\mathrm{Ca})$ to $\mathrm{n}(\mathrm{P})$ reached 1.67 (HAP-1, HAP2 , and HAP-3) and 1.50 (HAP-7). The $\mathrm{pH}(7.5,8.5,9.5$, and 10.5 ) of the obtained solution was adjusted by adding varying amounts of $\mathrm{NH}_{3} \cdot \mathrm{H}_{2} \mathrm{O}$ (HAP-4, HAP-5, HAP-6, and HAP-2). After stirring for $10 \mathrm{~h}$ at room temperature, the slurry was filtered, washed, and air-dried, and then the resulting white powders were obtained.

The influence of the initial $\mathrm{pH}$ on HAP morphology was investigated under the condition of a fixed amount of $\mathrm{HG}$ ( $5 \mathrm{wt} \%$ ) and the ratio of $\mathrm{nCa} / \mathrm{P}(1.67)$. The effects of $\mathrm{HG}$ were studied by fixing the initial $\mathrm{pH}$ at 10.5 and the $\mathrm{nCa} / \mathrm{P}$ ratio at 1.67. Finally, for the experiments designed to investigate the effects of the $\mathrm{nCa} / \mathrm{P}$ ratio $(1.50,1.67)$, the initial $\mathrm{pH}$ was set at 10.5 , and the amount of $\mathrm{HG}$ added was $5 \mathrm{wt} \%$. As a comparison, a sample prepared without HG under similar conditions was synthesized. In order to remove hydrolyzed gelatin, HAP-2 was calcined at $700^{\circ} \mathrm{C}$ as the control sample
TABLE 1: Experimental parameters for preparing HAP samples.

\begin{tabular}{lcccc}
\hline Sample & $\begin{array}{c}\mathrm{HG} \\
(\text { wt } \%)\end{array}$ & $\begin{array}{c}\mathrm{Ca}^{2+} / \mathrm{P}^{3+} \\
(\text { molar ratio })\end{array}$ & $\mathrm{pH}$ & $\begin{array}{c}\text { Calcined at } \\
700^{\circ} \mathrm{C}\end{array}$ \\
\hline HAP-1 & 2 & $5: 3$ & 10.5 & No \\
HAP-2 & 5 & $5: 3$ & 10.5 & No \\
HAP-3 & 10 & $5: 3$ & 10.5 & No \\
HAP-4 & 5 & $5: 3$ & 7.5 & No \\
HAP-5 & 5 & $5: 3$ & 8.5 & No \\
HAP-6 & 5 & $5: 3$ & 9.5 & No \\
HAP-7 & 5 & $3: 2$ & 10.5 & No \\
HAP-8 & 5 & $5: 3$ & 10.5 & Yes \\
\hline
\end{tabular}

(HAP-8). Table 1 summarizes the experimental parameters for preparing the various HAP samples in this study.

2.2. Characterization. The samples were characterized using scanning electron microscope (SEM; HITACHI S-4700), transmission electron microscope (TEM; JEM-100CX), Xray diffractometry (XRD; D/Max2500VB2+/PC; Cu-Ka radiation at $40 \mathrm{kV}$ with an $\mathrm{X}$-ray diffractometer), and Fourier transform infrared spectrometer (FTIR; Nexus 670).

\section{Results and Discussion}

Crystalline structures of HAP nanoparticles with various HG contents were confirmed by XRD measurements as shown in Figure 1. The sample without $\mathrm{HG}$ exhibits different diffraction pattern from the standardized HAP. Poorly crystalline HAP phases formed as the HG content in the solution was greater than $5 \mathrm{wt} \%$. The X-ray diffraction pattern of HAP-2 evidenced a much higher intensity for the (211), (310), and (231) peaks 


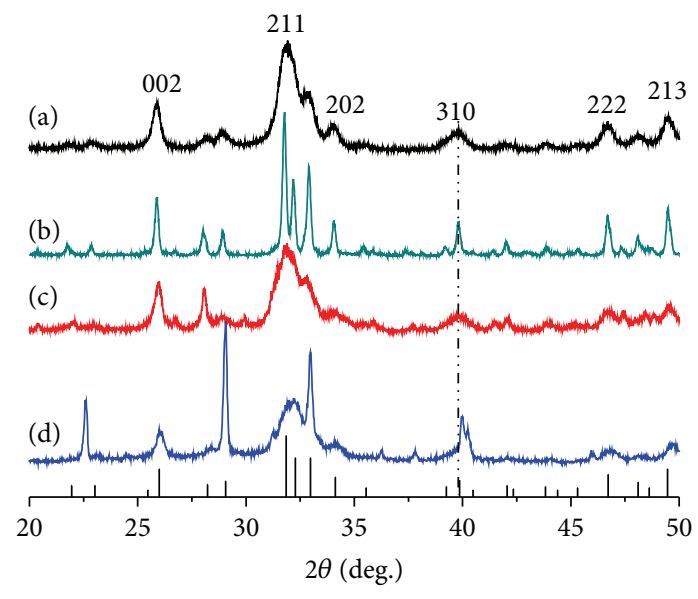

FIGURE 1: XRD patterns of (a) HAP-1, (b) HAP-2, (c) HAP-3, and (d) sample synthesized without $\mathrm{HG}$.

than the intensity of the HAP-1 peaks characteristic of HAP. This result can be attributed to a preferential growth of the HAP crystals in the (211), (310), and (231) directions due to the HG influence. However, with the increase of HG content, the formation of a large number of nuclei sufficiently depletes the concentration of calcium ions that are available for growth such that the crystals cannot grow very large. The preferred orientations of planes (002) and (310) were maintained in the sample series until HAP-2 and then fell precipitously as the HG content was increased to $10 \mathrm{wt} \%$. The formation and growth of HAP crystals were greatly influenced by the excess amount of HG. At the same time, it has also been found that the intensity of the peaks at $2 \theta \approx 28^{\circ}$ presents a rising tendency with the increase of HG content. It is well known that HAP contains two polymorphs: hexagonal and monoclinic. The hexagonal prism is related to the plane (102) $\left(2 \theta \approx 28^{\circ}\right)$ [12]. The result shows that HG can work well as a functional additive to promote the transformation of polymorphs. Therefore we speculate that when increasing the contents of HG, the monoclinic HAP polymorph would transform into the hexagonal one. These results indicate that the addition of HG is necessary for the synthesis of HAP.

The effect of $\mathrm{pH}$ changes on and crystallization behavior of HAP was evaluated using X-ray diffraction analysis (Figure 2). The characteristic peaks at $25.8^{\circ}, 31.8^{\circ}, 33.9^{\circ}$, and $39.8^{\circ}$ correspond to the (002), (211), (202), and (310) diffractions of the standard HAP crystallites, respectively. At the $\mathrm{pH}$ of 7.5, the HAP phase is directly observed on XRD pattern by adding HG. The increased XRD peak intensity implies that the crystallinity of HAP increases with the increase of the $\mathrm{pH}$ value. And the intensity of peaks at $2 \theta \approx$ $28^{\circ}$ presents decline tendency with the increase of the $\mathrm{pH}$ value. It shows that the polymorphs and the crystallinity of HAP can be changed by controlling the $\mathrm{pH}$ of the initial reaction, which results in altering the morphology and size of HAP.

We also synthesized pure HAP nanoparticles using an initial $\mathrm{Ca} / \mathrm{P}$ ratio of 1.50 . The XRD patterns (Figure 3 ) show the characteristic peaks similar to those of HAP-2. Normally,

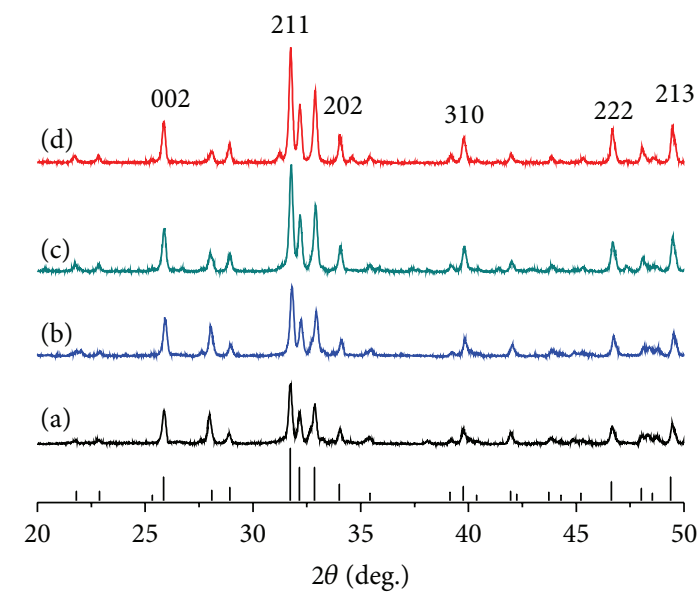

FIGURE 2: XRD patterns of the HAP with varying $\mathrm{pH}$ : (a) 7.5 , (b) 8.5 , (c) 9.5 , and (d) 10.5 .

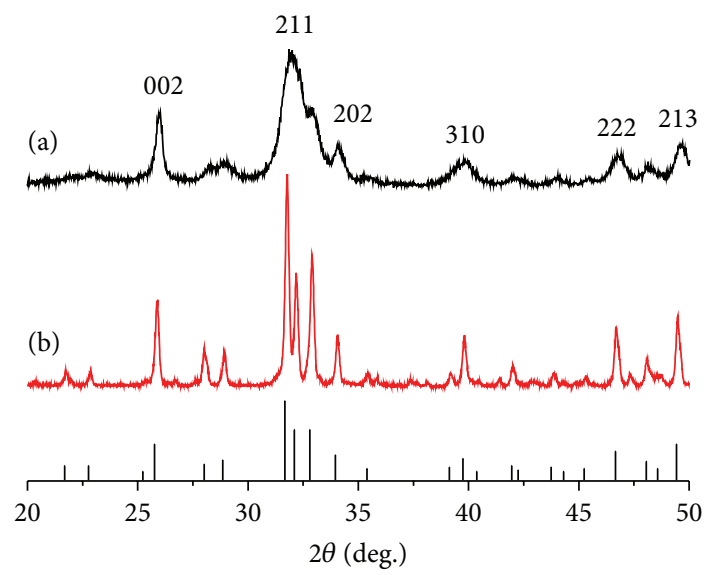

FIGURE 3: XRD patterns of products with varying $\mathrm{Ca} / \mathrm{P}$ molar ratio: (a) 1.50 (HAP-7) and (b) 1.67 (HAP-2).

without HG as an additive, HAP could not be synthesized with the $\mathrm{Ca} / \mathrm{P}$ ratio of 1.50 . The amorphous calcium phosphate (TCP) precursor could be prepared by conventional chemical precipitation methods without $\mathrm{HG}$ with the ratio of 1.50 (HAP-7). In this work, we found that the HG was able to promote the formation of HAP with initial $\mathrm{Ca} / \mathrm{P}$ molar ratio of 1.50 . The impurity phases were inhibited by adding HG, demonstrating HG's promotion of HAP formation. We speculate that $\mathrm{HG}$ can work well as a functional additive to reduce the transition enthalpy from TCP to HAP.

Figure 4(a) shows the FTIR spectra of the sample HAP-2 synthesized with HG. In order to further understand the structure of the components and formation process of HAP, the FTIR spectra of the HG (Figure 4(b)) and HAP8 (Figure 4(c)) are also shown as comparison. Both HAP-2 and HAP- 8 have the absorption bands at $1036 \mathrm{~cm}^{-1}, 565 \mathrm{~cm}^{-1}$, and $603 \mathrm{~cm}^{-1}$, confirming the presence of a phosphate group. Moreover, typical absorption bands for hydroxyl vibrational modes at $3572 \mathrm{~cm}^{-1}$ were also identified for both samples. It is noted that HAP-2 has the absorption band at $1638 \mathrm{~cm}^{-1}$, which is due to the presence of $\mathrm{C}=\mathrm{O}$ in $\mathrm{HG}$ and a broad 

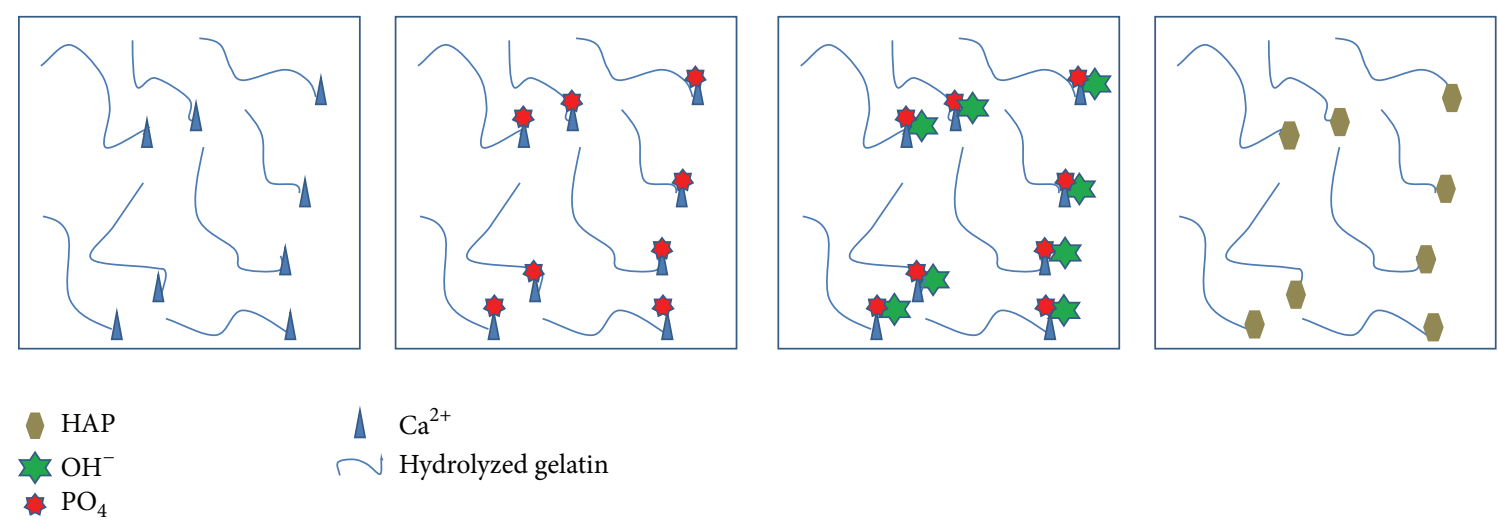

Scheme 2: Possible mechanisms of HAP formation facilitated by hydrolyzed gelatin as an additive.

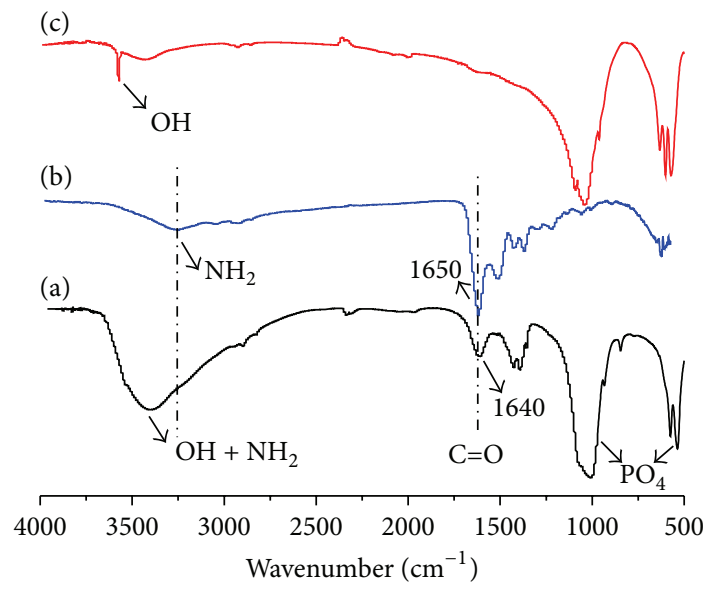

Figure 4: FTIR spectra of (a) HAP-2, (b) HG, and (c) HAP-8.

absorption band at $3572 \mathrm{~cm}^{-1}$ which represents overlapping peaks of amido and hydroxyl groups. Furthermore, the bands at $1421 \mathrm{~cm}^{-1}$ and $1456 \mathrm{~cm}^{-1}$ can be attributed to the existence of carboxylate, confirming the chemical bonding between HG and calcium ions. Compared to HAP-2, there are no absorption bands of $\mathrm{C}=\mathrm{O}$ and carboxylate in HAP- 8 , which is the result of the removal of $\mathrm{HG}$ after calcination. Comparing with HAP-2 and HAP-8, the red shift of $1640 \mathrm{~cm}^{-1}$ band in HG was used to confirm the chemical bond formation between carboxyl ions in HG and HAP. It is believed that the red shift in the HAP-2 system is encouraged by the wagging vibration through the covalent bond formation with $\mathrm{Ca}^{2+}$ ions of HAP nanocrystals. Overall, the FTIR results are consistent with the XRD results.

The elemental composition and microstructure of HAP-2 and HAP-8 were investigated using EDS and TEM (Figure 5). From the EDS analysis, we can easily identify the existence of oxygen, carbon, calcium, and phosphorus atoms in both HAP-2 (Figure 5(a)) and HAP-8 (Figure 5(b)). The relative ratio of calcium to phosphorus $(\mathrm{Ca} / \mathrm{P})$ is approximately 1.62 , which is close to the initial set value of 1.67 .
The calcium deficiency may be due to the EDS measurement error or caused by the imperfect crystal lattice and the presence of additional phases. TEM images show that HAP-2 (Figure 5(c)) nanoparticles are generally spherical and the particles size is approximately $40-60 \mathrm{~nm}$ in diameter. Compared to HAP-2 (Figure 5(c)), the particle size of HAP8 (about 30-100 nm, see Figure 5(d)) increased after the removal of HG on the surface, suggesting that HG also played an important role in the particle size control during the synthesis of HAP.

Our findings may be explained by the molecular structure of the added gelatin. The molecular chain of hydrolyzed gelatin is composed of a series of amino acids and their telopeptides consist of many functional groups, such as carboxyl group, hydroxyl oxygen atom, and oxygen atoms from hydroxyl and carbonyl groups. Scheme 2 shows the schematic representation of HAP microcrystal formation via terminal carboxyl group. As the calcium ions accumulate near the terminal carboxyl group and interact with it, thus, terminal carboxyl group must gain an overall positive charge. These positively charged areas may be attracted to negatively charged phosphate ions $\left(\mathrm{PO}_{4}\right)^{3-}$, initiating the nucleation of HAP particles on the terminal of hydrolyzed gelatin chain $[11,13,14]$. The HG solution can provide more terminal carboxyl group for the selective attraction of calcium ions, which facilitates the nuclei formation of HAP. Moreover, HG can serve as a good dispersant to inhibit the conglomeration of HAP [15].

\section{Conclusion}

In contrast to conventional high temperature synthesis, we developed a synthesis protocol of the nanohydroxyapatite at room temperature using a hydrolyzed gelatin as an additive. The results demonstrated that the HG can promote the formation of HAP, even in the calcium-deficient case $(\mathrm{Ca} / \mathrm{P}$ molar ratio of 1.50), and act as a dispersant and structure modifier as well. The amount of HG and the initial $\mathrm{pH}$ of the precursor reactants greatly influenced the nucleation and growth of HA nanocrystals. The as-synthesized HAP has uniformly spherical structure and an average particle size of 


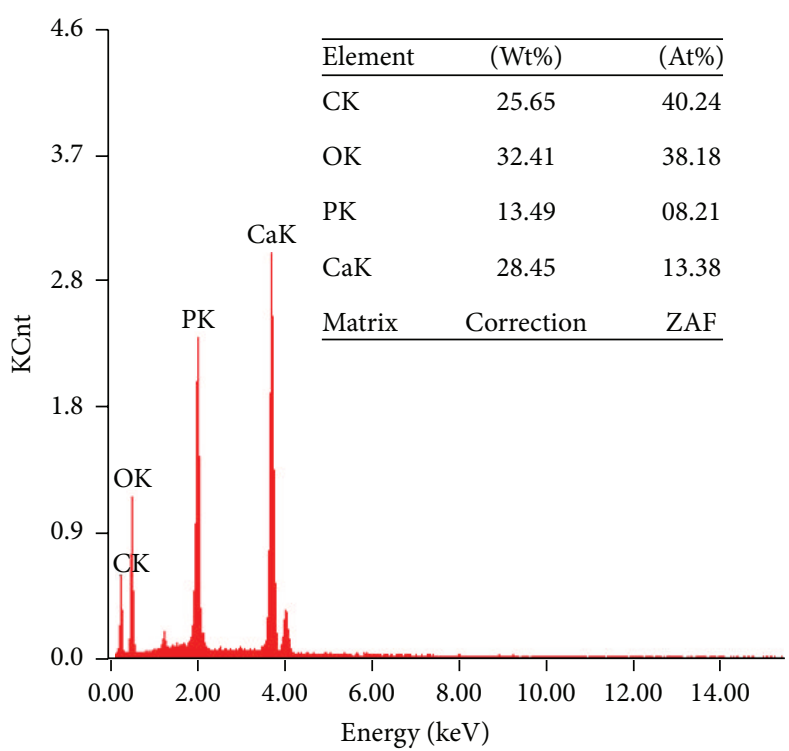

(a)

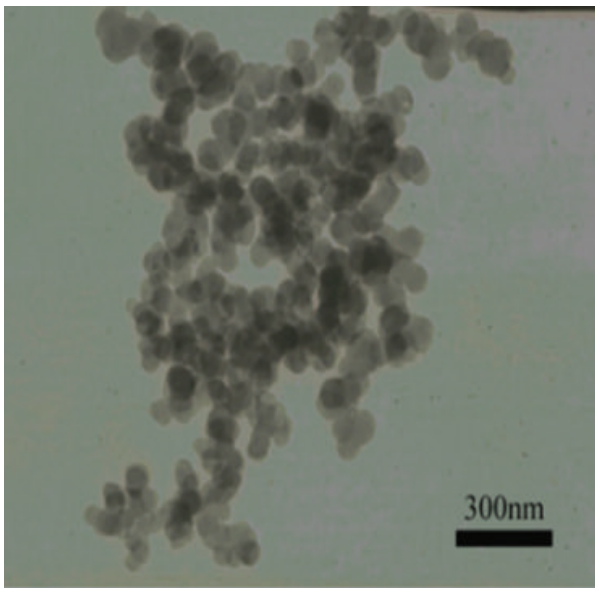

(c)

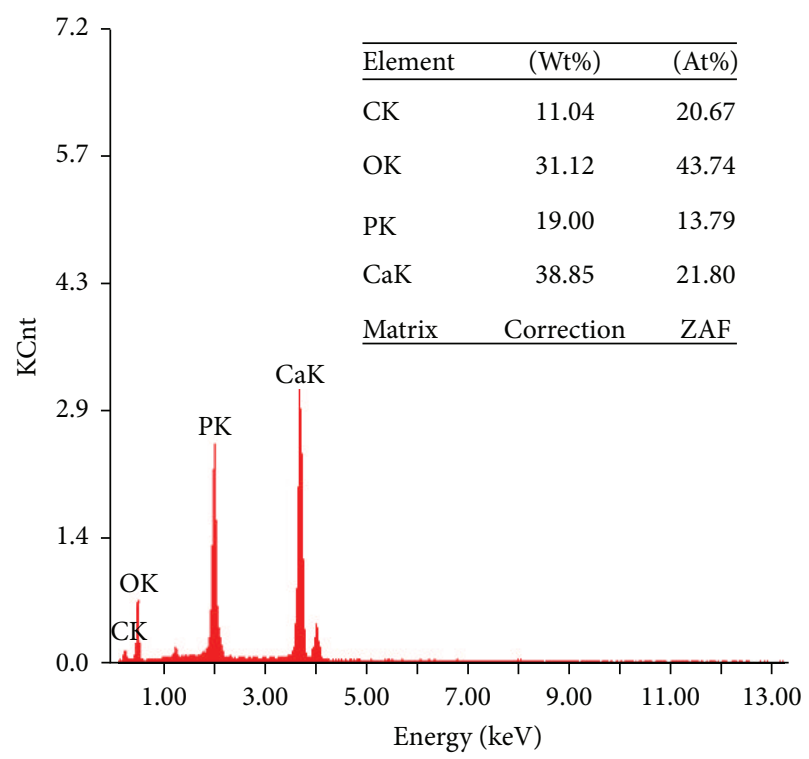

(b)

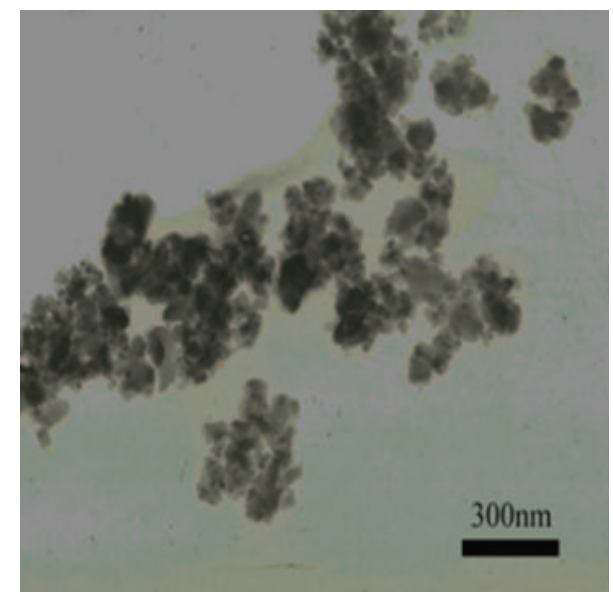

(d)

Figure 5: EDS and TEM images of (a, c) HAP-2 and (b, d) HAP-8.

about 40-60 $\mathrm{nm}$. We believe the method described in this study is an efficient and facile strategy for the synthesis of well-dispersed nano-HAP.

\section{Disclosure}

Ding Ding and Tengfei Yu are co-first authors.

\section{Conflict of Interests}

The authors declare that there is no conflict of interests regarding the publication of this paper.

\section{Authors' Contribution}

Ding Ding and Tengfei Yu contributed equally to this work.

\section{Acknowledgment}

Financial support from the National Science Foundation of China (nos. 51272017 and 51432003) is gratefully appreciated.

\section{References}

[1] S. Ramakrishna, J. Mayer, E. Wintermantel, and K. W. Leong, "Biomedical applications of polymer-composite materials: a review," Composites Science and Technology, vol. 61, no. 9, pp. 1189-1224, 2001.

[2] S. Singh, R. M. Kumar, K. K. Kuntal et al., "Sol-gel derived hydroxyapatite coating on $\mathrm{Mg}-3 \mathrm{Zn}$ alloy for orthopedic application," JOM: The Journal of the Minerals, Metals \& Materials Society, vol. 67, no. 4, pp. 702-712, 2015.

[3] V. Sternitzke, M. Janousch, M. B. Heeb, J. G. Hering, and C. A. Johnson, "Strontium hydroxyapatite and strontium carbonate as templates for the precipitation of calcium-phosphates in the 
absence and presence of fluoride," Journal of Crystal Growth, vol. 396, pp. 71-78, 2014.

[4] A. Sharma, S. Payne, K. S. Katti, and D. R. Katti, "Evaluating molecular interactions in polycaprolactone-biomineralized hydroxyapatite nanocomposites using steered molecular dynamics," JOM, vol. 67, no. 4, pp. 733-743, 2015.

[5] M. Yang, Y. Shuai, C. Zhang et al., "Biomimetic nucleation of hydroxyapatite crystals mediated by Antheraea pernyi silk sericin promotes osteogenic differentiation of human bone marrow derived mesenchymal stem cells," Biomacromolecules, vol. 15, no. 4, pp. 1185-1193, 2014.

[6] C.-M. Xie, X. Lu, K.-F. Wang et al., "Silver nanoparticles and growth factors incorporated hydroxyapatite coatings on metallic implant surfaces for enhancement of osteoinductivity and antibacterial properties," ACS Applied Materials and Interfaces, vol. 6, no. 11, pp. 8580-8589, 2014.

[7] X.-J. Zhang, D.-Y. Lin, X.-H. Yan, and X.-X. Wang, "Evolution of the magnesium incorporated amorphous calcium phosphate to nano-crystallized hydroxyapatite in alkaline solution," Journal of Crystal Growth, vol. 336, no. 1, pp. 60-66, 2011.

[8] K. S. Vecchio, X. Zhang, J. B. Massie, M. Wang, and C. W. Kim, "Conversion of bulk seashells to biocompatible hydroxyapatite for bone implants," Acta Biomaterialia, vol. 3, no. 6, pp. 910-918, 2007.

[9] M. Kester, Y. Heakal, T. Fox et al., "Calcium phosphate nanocomposite particles for in vitro imaging and encapsulated chemotherapeutic drug delivery to cancer cells," Nano Letters, vol. 8, no. 12, pp. 4116-4121, 2008.

[10] X. J. Wang, Y. B. Li, J. Wei, and K. B. De Groot, "Development of biomimetic nano-hydroxyapatite/poly(hexamethylene adipamide) composites," Biomaterials, vol. 23, no. 24, pp. 47874791, 2002.

[11] M. T. Jahromi, G. Yao, and M. Cerruti, "The importance of amino acid interactions in the crystallization of hydroxyapatite," Journal of The Royal Society Interface, vol. 10, no. 80, Article ID 20120906, 2013.

[12] D. Aquilano, M. Bruno, M. Rubbo, F. R. Massaro, and L. Pastero, "Low symmetry polymorph of hydroxyapatite. theoretical equilibrium morphology of the monoclinic $\mathrm{Ca}_{5}(\mathrm{OH})\left(\mathrm{PO}_{4}\right)_{3}$," Crystal Growth and Design, vol. 14, no. 6, pp. 2846-2852, 2014.

[13] F. Nudelman, K. Pieterse, A. George et al., "The role of collagen in bone apatite formation in the presence of hydroxyapatite nucleation inhibitors," Nature Materials, vol. 9, no. 12, pp. 10041009, 2010.

[14] B. Palazzo, D. Walsh, M. Iafisco et al., "Amino acid synergetic effect on structure, morphology and surface properties of biomimetic apatite nanocrystals," Acta Biomaterialia, vol. 5, no. 4, pp. 1241-1252, 2009.

[15] R. Palanivelu and A. R. Kumar, "Synthesis, characterization, in vitro anti-proliferative and hemolytic activity of hydroxyapatite," Spectrochimica Acta Part A, vol. 127, pp. 434-438, 2014. 

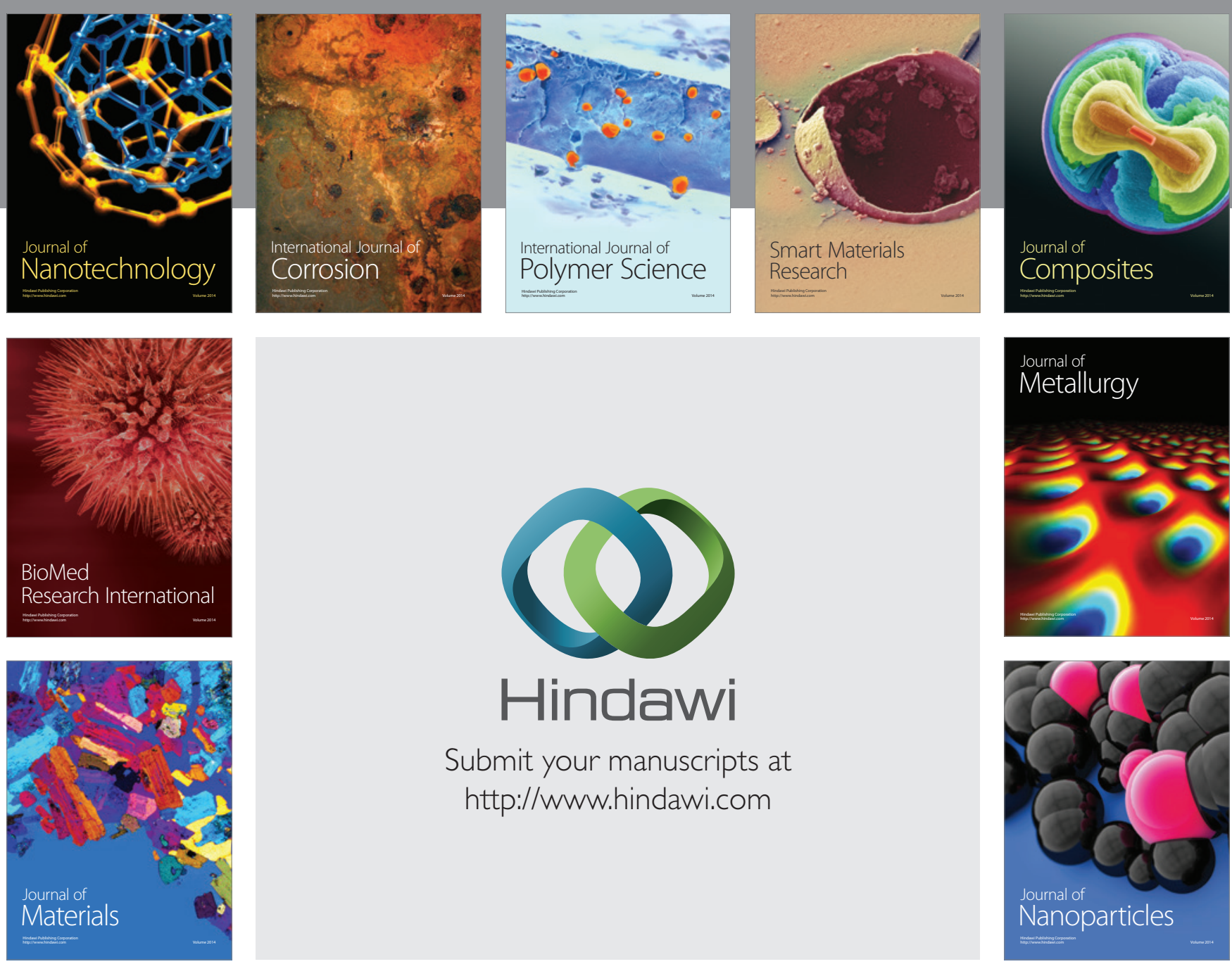

Submit your manuscripts at http://www.hindawi.com
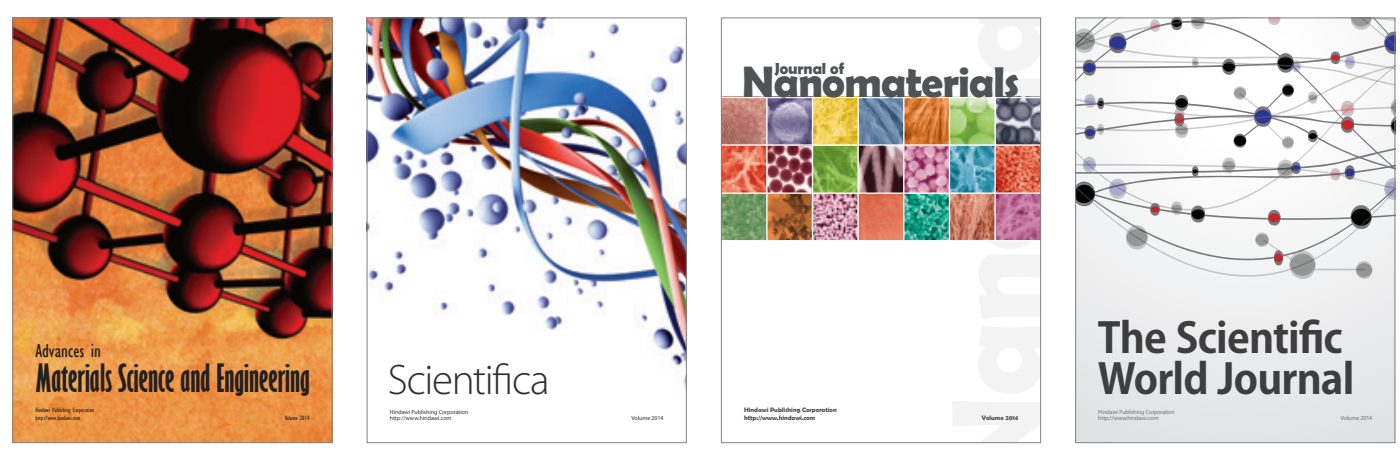

\section{The Scientific World Journal}
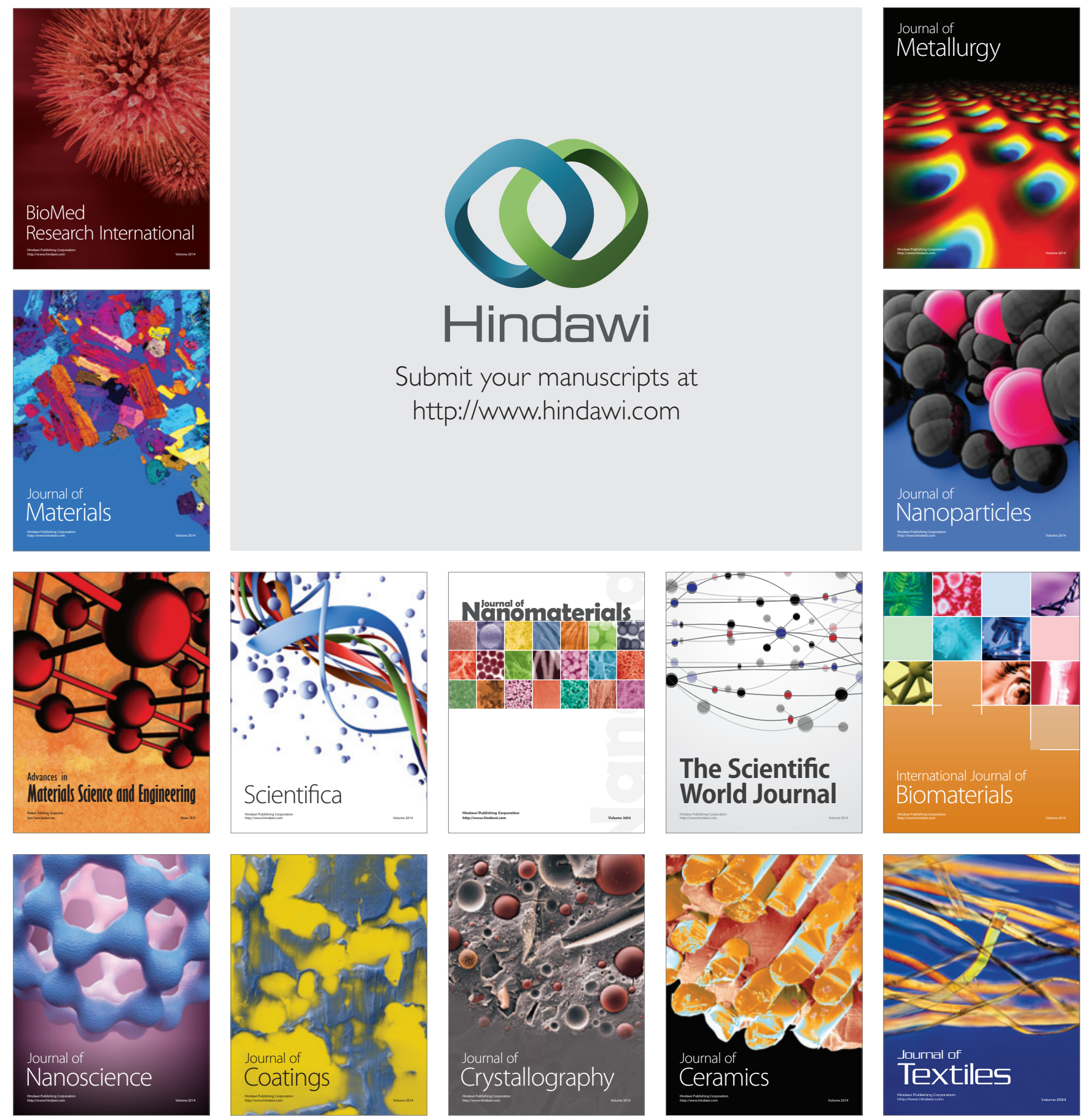